\title{
استراتيجيات تصميم المدن المستدامة باستخدام تقتية نمذجة معلومات البناء BIM
}

\section{أ.د محمد هثام سعودي 1 ، أ.د هثام جلال الشيمي 2 ، د.محمد أحمد فؤاد مهدي 3 م. م شيماء فتحي عاشور 4}

أستاذ العمارة ـ قسم العمارة ـ كلية الفنون الجميلة -جامعة الاسكندرية - العمارية

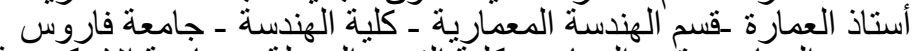

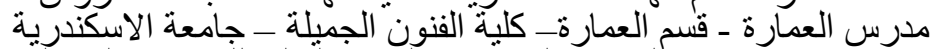

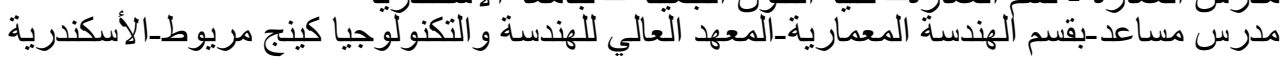

إن الإستعانة بتطبيقات التكنولوجيا الحديثة والنماذج الرقمية في العمارة إنتقلت بالتصميم إلي مراحل متقدمة من الابداع

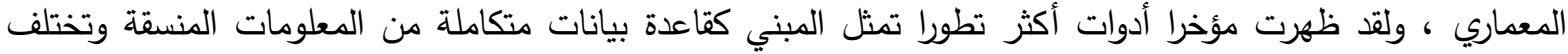

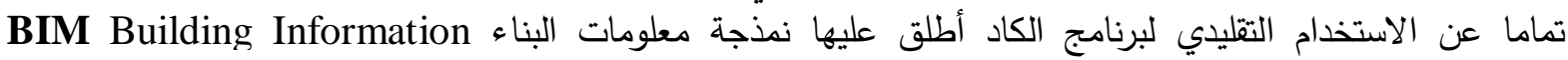

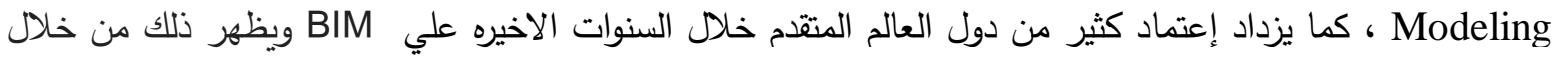

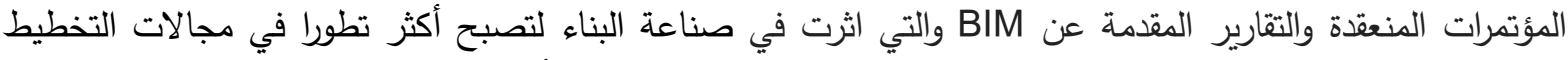

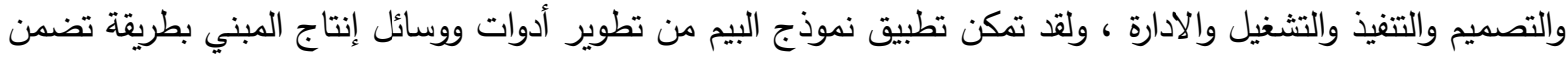

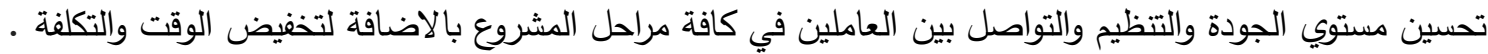

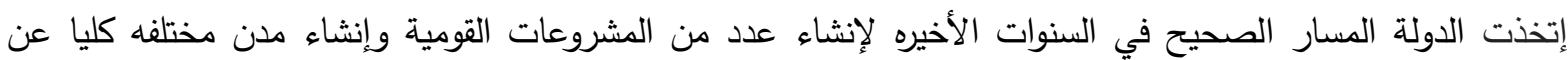

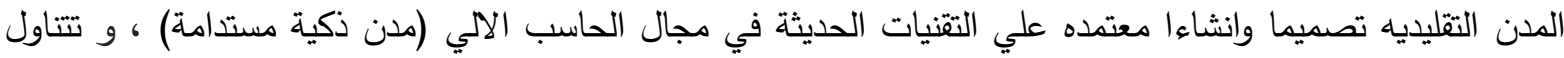

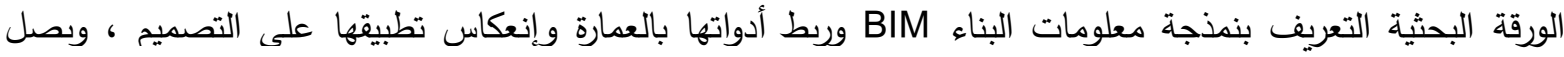
البحث إلى استراتيجية للتطبيق داعمة لتحقيق مدن خضراء مستدامة من خلال توثيق و وإبراز دور التطبيقات التبات الحديثة في مجال الحاسب الالي وتأثير ذلك علي جودة المنتج المعماري.

الكلمات الدالة: نمذجة معلومات البناء ,المدن الخضراء, المشاريع القومية بمصر ,الاستدامة .

المقدمة

ظهرت في الفترة الأخيرة تقنيات حديثة ومتطورة في إدارة المشاريع والتي تعد مرحلة هامة للانتقال لمستقبل اكثر ازدهارا

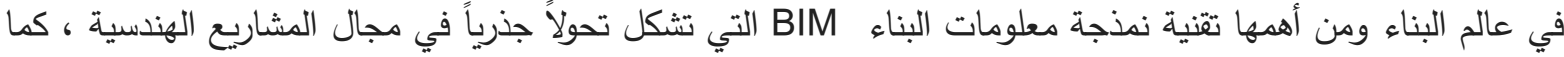

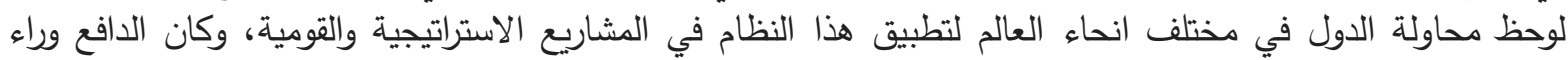

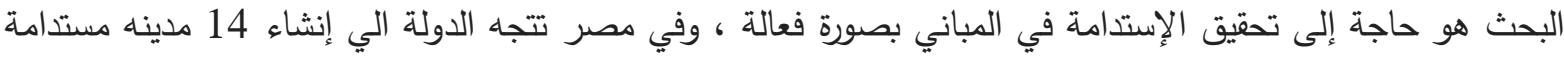

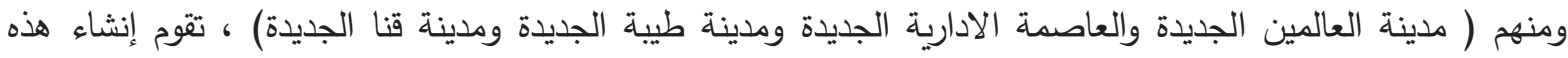

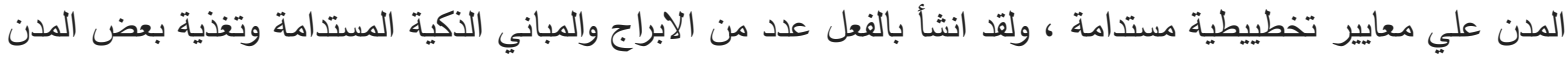
بمحطات تخزين الطاقة الثمسية مختلفة القدرات ، ويستوجب التفاعل الملحوظ من قبل المصات التصمين لدعم هذه المبادرات

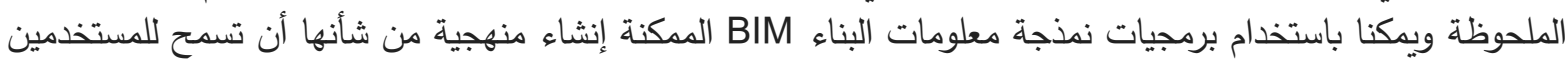
بسهولة تطوير نظام للتطبيق والتقييم لإستخدامهم الخاص في بيئتهم وتمكين فريق العمل من إتخاذ وتحسين القرارات خلال عملية التصميم والتحرر من التقيد بالثروط المعطلة المرتبطة بالبناء التقليدي.

إن تصور الآلية المتبعة في أساليب القياس عبر مجموعة متباينة من العاملين بالبناء من الصعب تحتيك تحقيقها عندما يتم تقييد

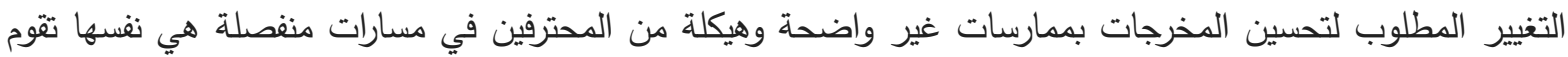

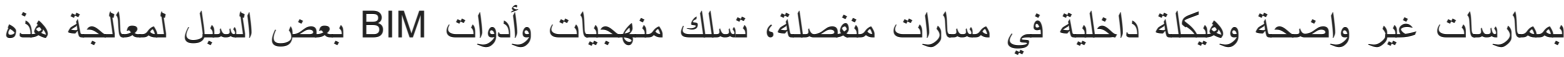

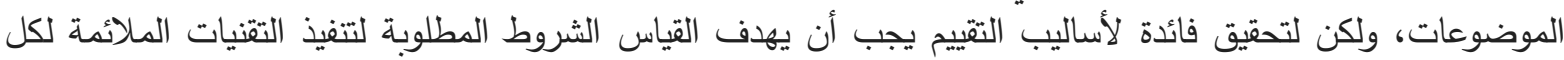

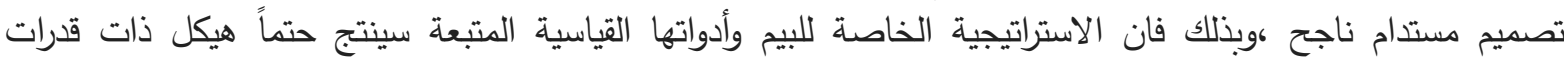
تنظيمية بشكل أفضل وبعدها ستكون قيمة مضافة في مجال الاستدامة. الهوف البحثي

تهدف الورقة البحثية إلي وضع استراتيجية لتصميم ددن مستدامة وذلك باستخدام نمذجة معلومات البناء

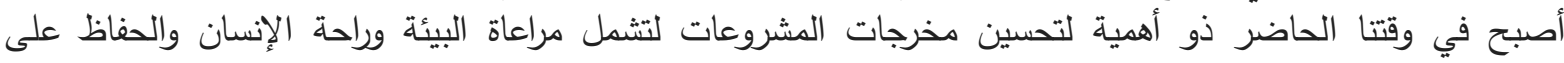

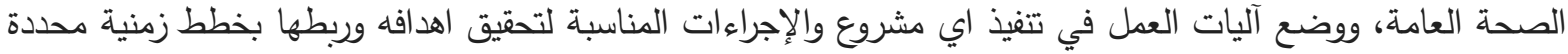

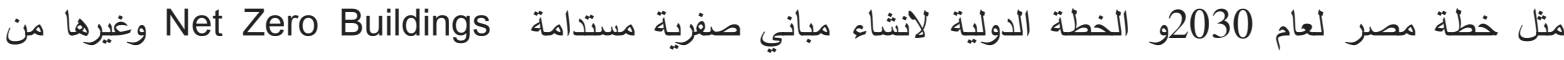


الخطط والمبادرات،التي تساهم في التكامل بين التخصصات المختلفة ذات الصلة بإدارة عمليات التصميم والبناء والتشغيل والصيانة. 1. ماهية المدن المستدامة، أو المدينة البيئية وهى مدن الجيل الرابع والتىى يتوجه لانشائها جميع دول العالم المتقدم لإدراكهح

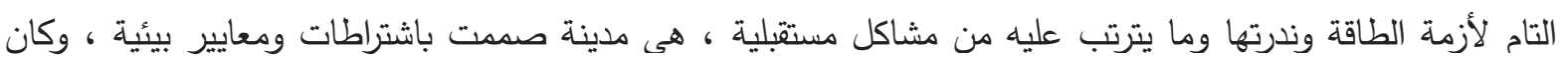

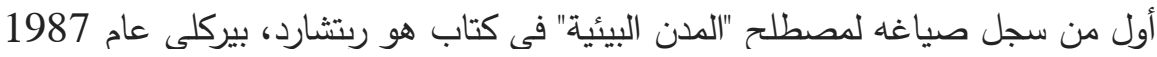

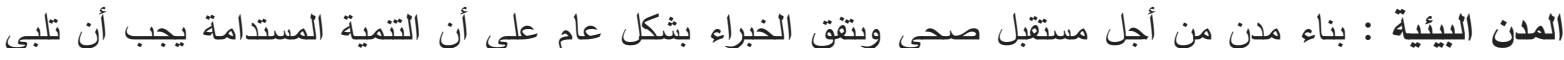

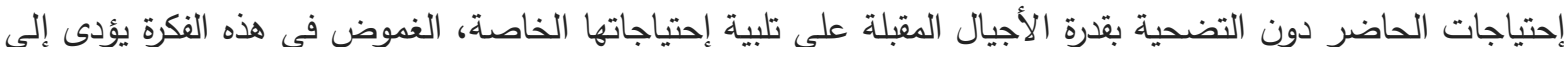

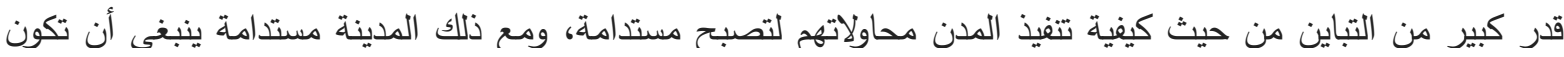

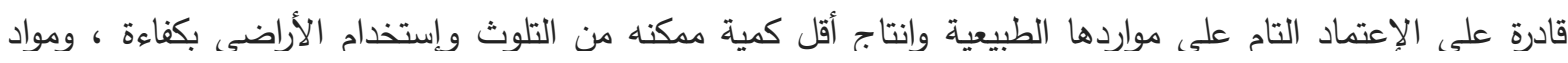

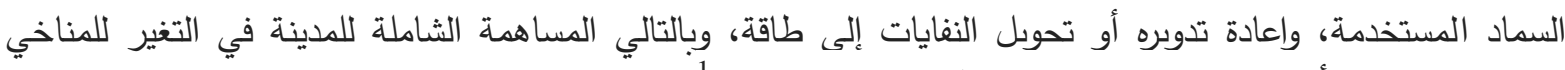
سيكون في الحد الأدنى، إذا ما إنضمت إدور إلى مثل هذه الممارسات. 1

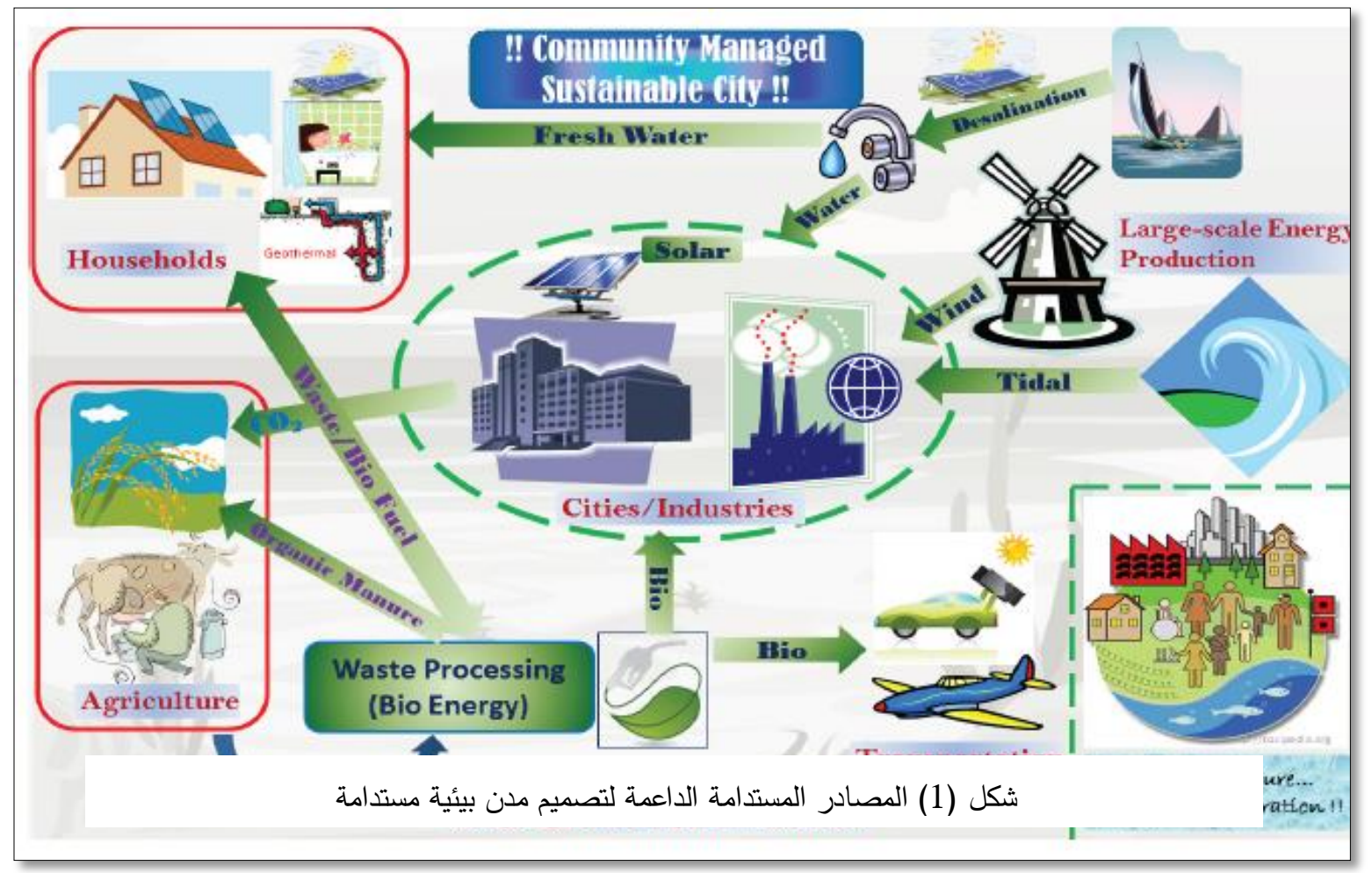

وتشير التقديرات إلى أن أكثر من 50٪ من سكان العالم يعيشون الآن فى الددن والمناطق الحضربة هذه المجتمعات

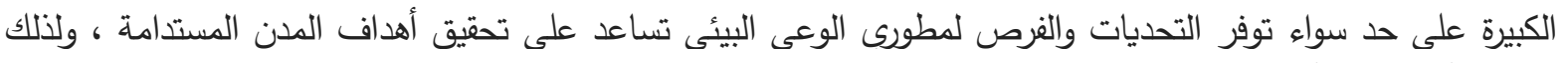

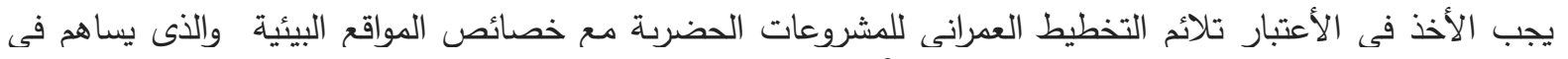

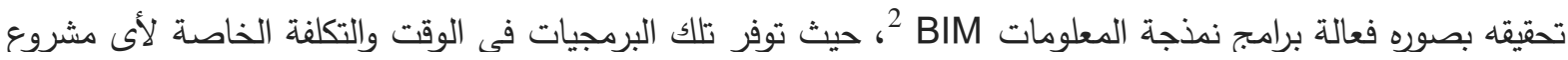

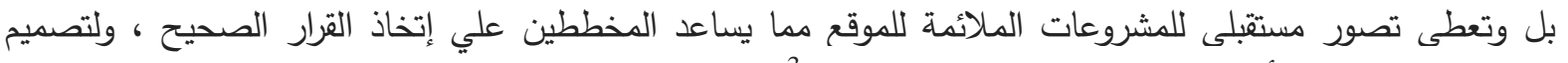

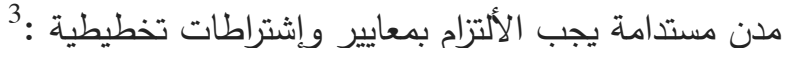
1- الهيكلة المستدامة من خلال دراسة وتوفير البنية التحتية لسير المدينة، مع التزام بمعايير تحقيق العمارة المستدامة

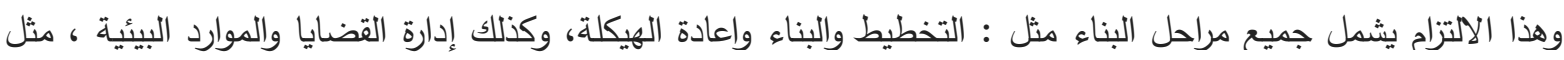

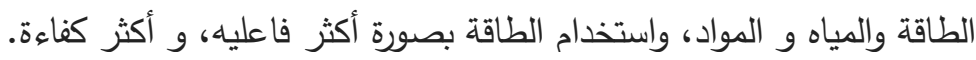

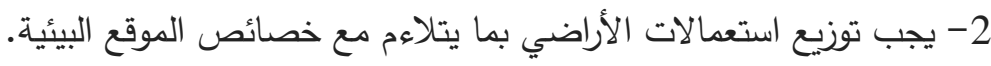

3- تحديد الفراغ الملائم والمناسب لمختلف أنواع التتمية الموجودة أو المتوقع تواجدها في المنطقة بحيث تحافظ على البيئة الطبيعية والأماكن الأكثر حساسية بيئيا. 
4- تأمين الموقع وحمايته من الكوارث الطبيعية المحتملة وخاصة السيول والفيضانات والحرائق وهبوط وإنزلاق التربة في المنطقة المقترحة.

5- التوزتح المكانى لإشغالات الأراضى من كتل سكنية وعلاقتها بالمناطق الخضراء والمفتوحة وكذلك علاقتها بالتلوث

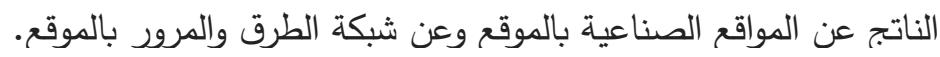

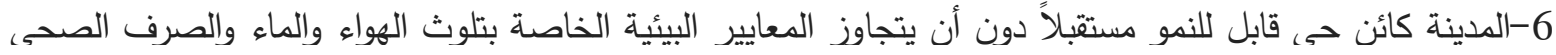
والمخلفات الصناعية لذا يجب التقييم البيئي المستمر والمتواصل لمعرفة مدى إمكانية المشروع في السير قدماً نحو

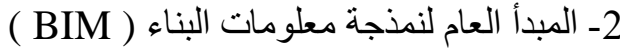

نمذجة معلومات البناء (BIM) هي واحدة من أهم التطورات الأخيرة في مجالات الهندسة المختلفة باستخدام

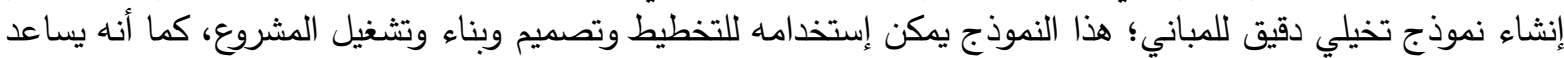

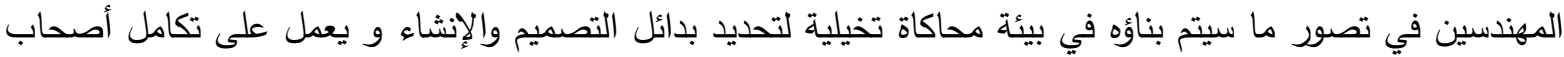

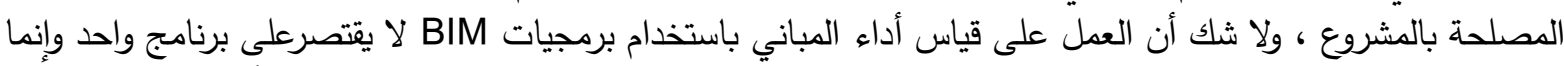

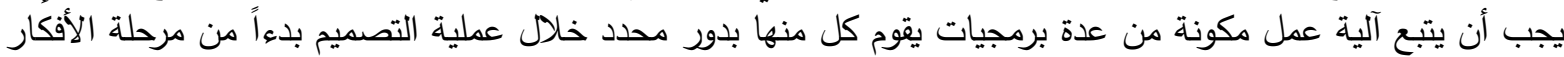

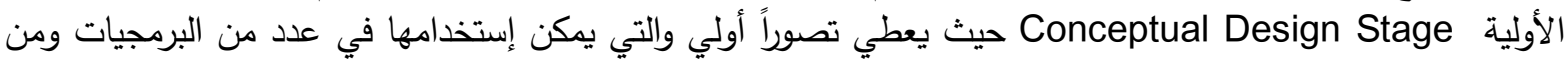

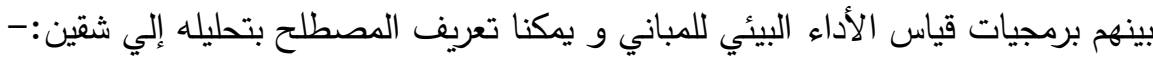

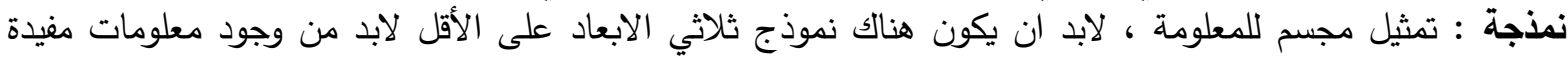

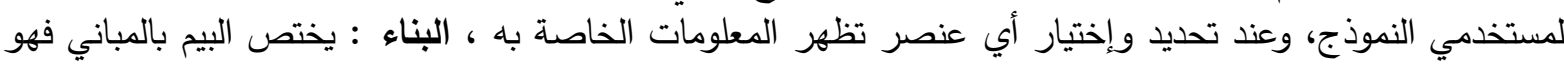
ليس عام مثل تقنية الكاد يمكن رسم كل شيء به بل هو متخصص التئ ، و و هو يستخدم أثناء عملية التصميم و التنفيذ و لئاء

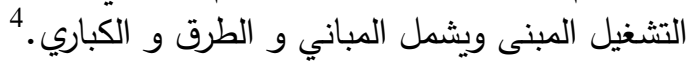
نمذجة معلومات البناء BIM : هي منهجية مبتكرة تساعد في تحسين الاتصال والتعاون بين الجهات المعنية في مشروع

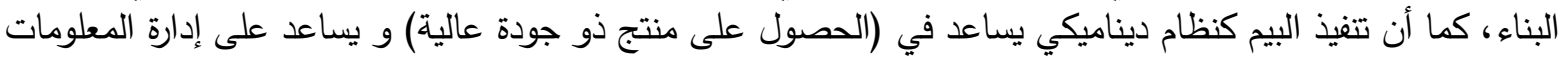
خلال دورة حياة المشروع، ليس فقط أثناء التصميم والبناء ولكن أيضا أثناء الثناء التشغيل والصيانة.
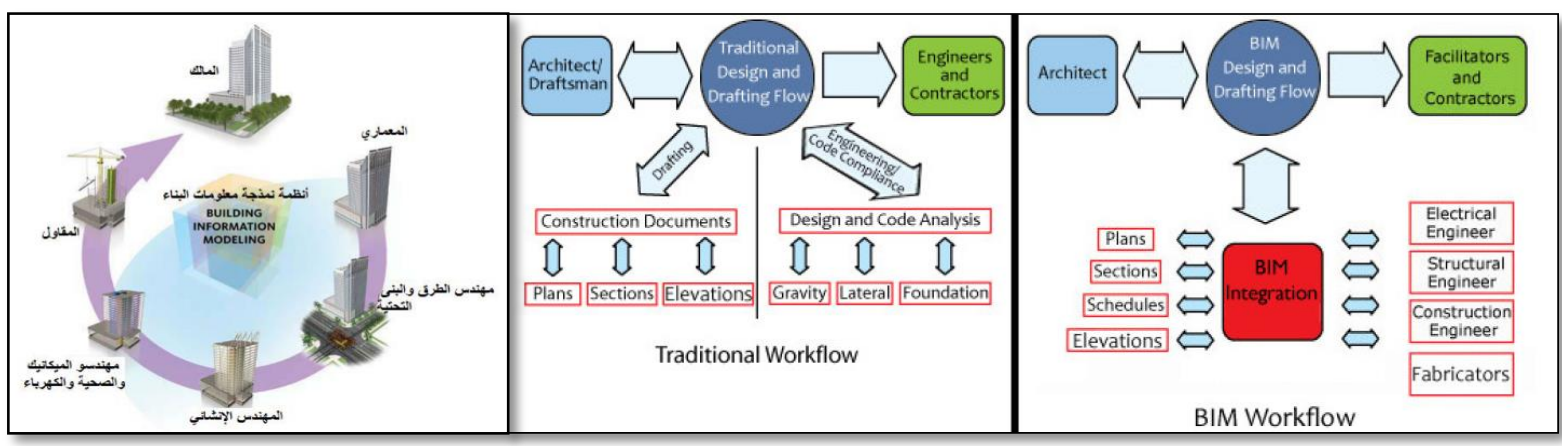

$$
\text { شكل(2) تكامل جميع التخصصات الهندسية ومسئولين المشروع لانشاء نموذج لبناء }
$$

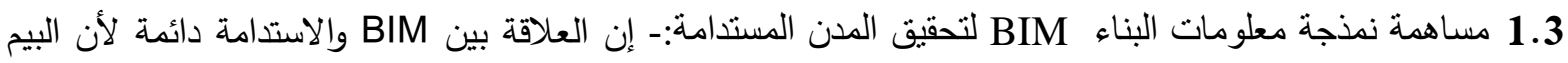

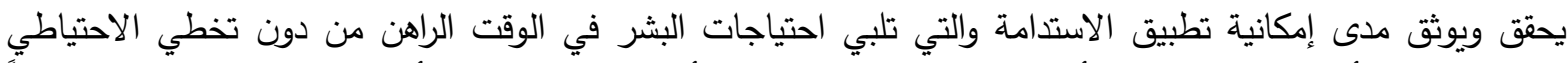

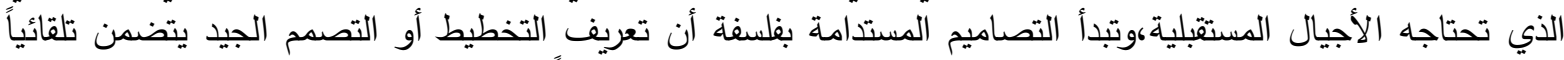

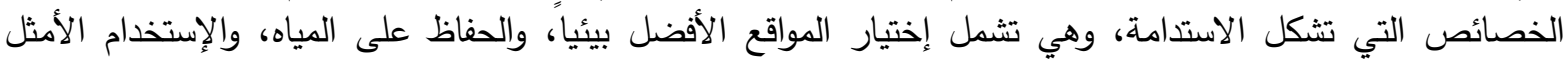

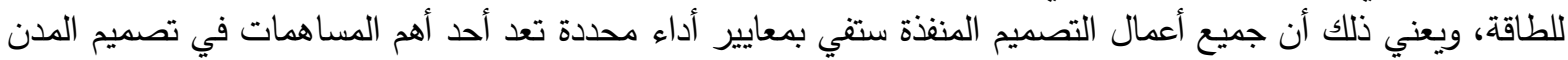

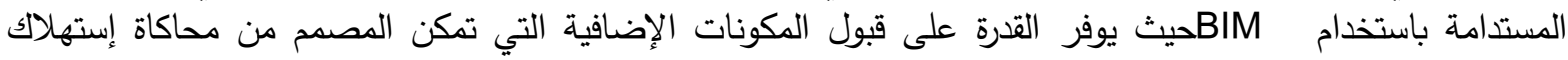

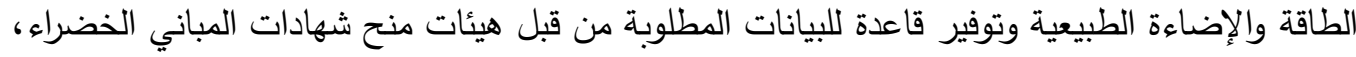



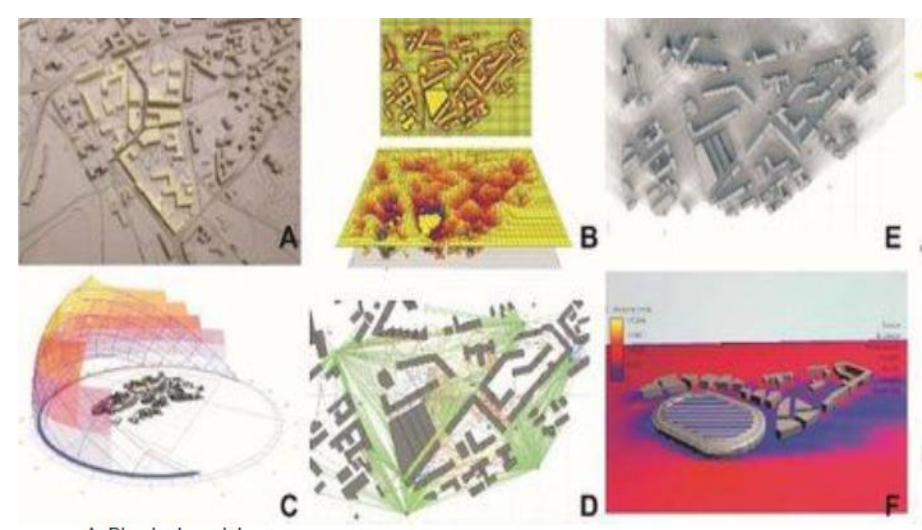

E

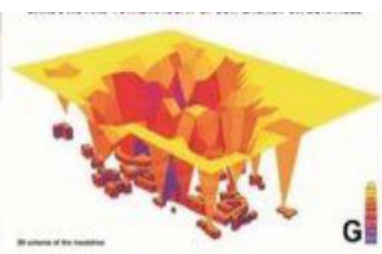

B. Model transposition to the software

C. Sunlight analysis on surfaces determine the amount of passive energy,

D. Sound/noise analysis,

E. Analysis of the year shadow range,

F. Wind analysis,

$\mathrm{G}$. Shadow analysis taking into account the annual passive energy distribution,

H. Colorful of the shadow range.

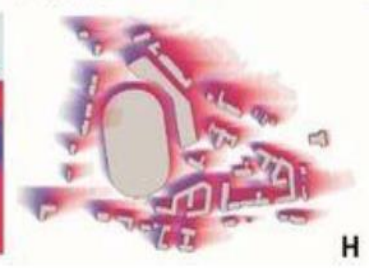

- نموذج تخطيطي للموقع ونموذج متوقع لحركة المواصلات

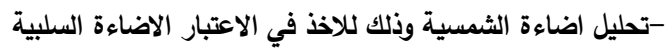

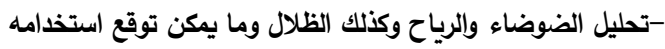
من عناصر للاظلال وتعطي برمجيات المحاكاه الوان للاضاءة والظلال

شكل(3) خطوات تصميم مدن مستدامة باستخدام برامج

أيضا باستطاعة BIM أن يجعل من السهل تحديد الموقع الأمثل وتوجيه المباني لتحقيق أقصى قدر من توليد الطاقة المتجدة والحد من استهلاك الطاقة.

4. تطبيق نمذجة معلومات البناء BIM في تصميم المدن المستدامة بمصر عند تطبيق تقنية المدن الذكية المستدامة يظهر مصطلح نمذجة المعلومات المدنية؛ والهدف منه إنثاء وصيانة البنية

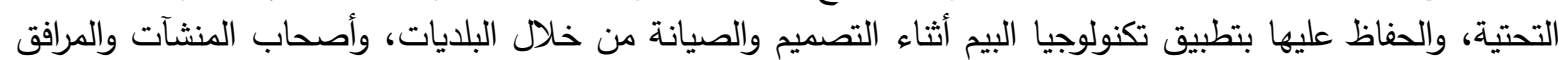

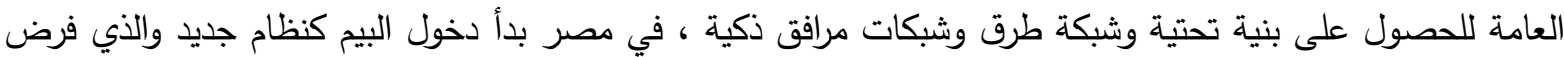

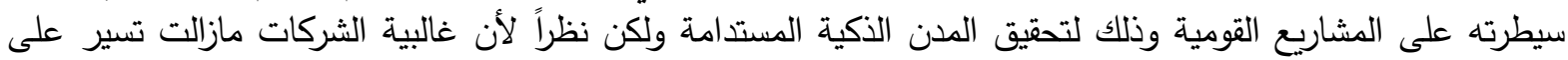

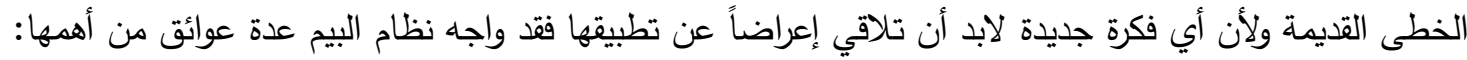

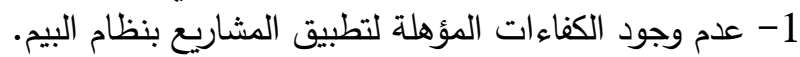
2-حاجة الثركات لأجهزة حديثة ومتطورة.

3- غالبية الشركات دائماً تنظر إلى تحقية الثيق الدكاسب بأقل التكاليف

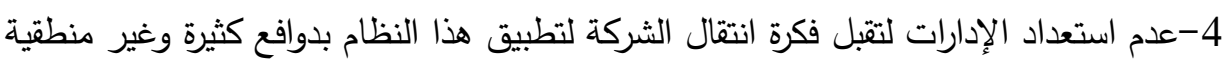

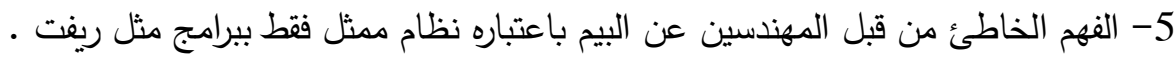

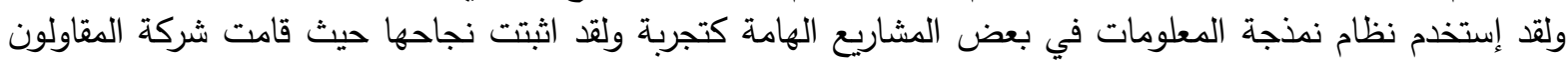

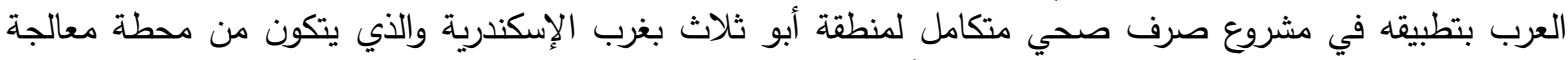

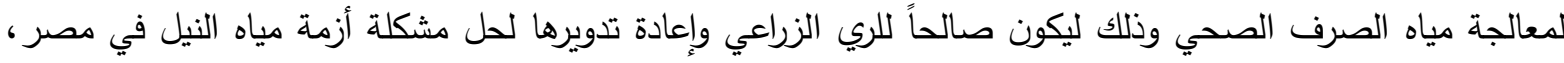

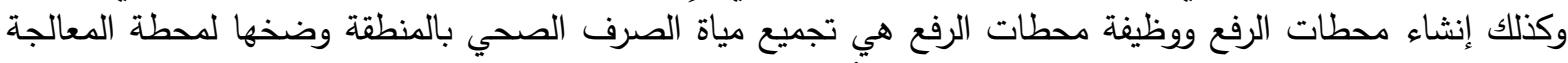
بطلمبات هيدروليكية وبتطبيق البيم تم حل عدة مشاكل أهمها:

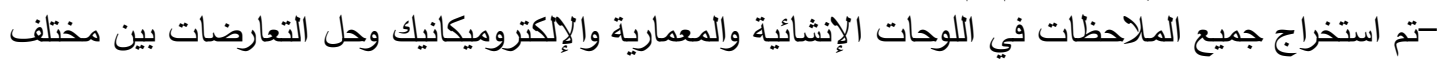
التخصصات المدنية قبل التنفيذ.

-تم عمل حصر للأعمال الخرسانية وحديد التسليح وذلك لعمل المقايسات. -سهولة فهم المنشأ وكيفية عمله ومن ثم استخراج خطوات التتفيذ بشكل أسهل وذلك لأن أن محطات المعالجة معقدة في تنفيذها. وبالفعل إتجهت الدولة بالفعل بتصميم وإنشاء عدد من المدن الجديدة المستدامة التي تقوم علي أسس و معايير تخطيطية

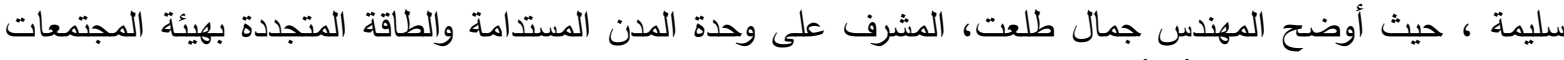

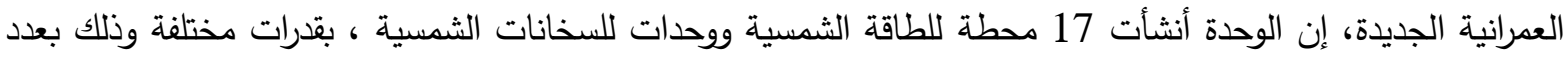
من المدن الجديدة ( مدينة طيبة الجديدة - مدينة العاصمة الادارية الجديدة سمدينة قنا الجديدة - مدينة السادات ) ) 
أنه تم تتفيذ محطات للطاقة الثمسية ببعض المباني الحكومية والطرق الرئيسية بـ12 مدينة جديدة، بإجمالي قدرة 600 التبات

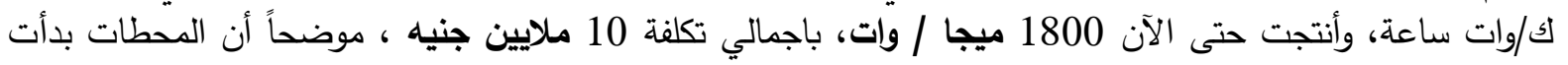

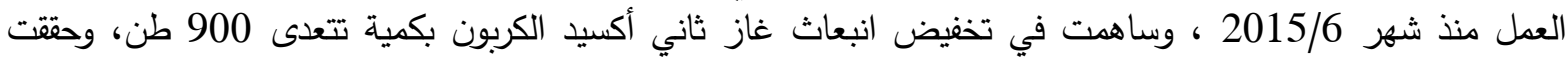

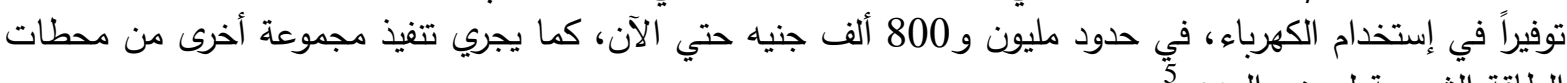

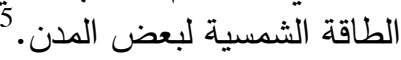

وفي مجال إعادة تدوير المياه، سيتم إعادة استخدام المياه الرمادية في 10 مدن جديدة، من خلال تتفيذ 10 وحدات وات التيات

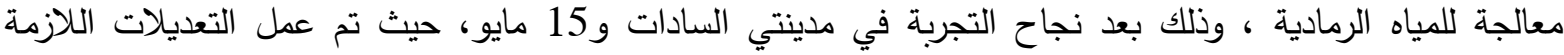

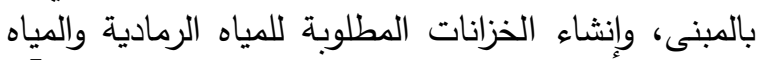
المعالجة، والاستفادة منها في ري المسطحات الخدات الخضراء. 7

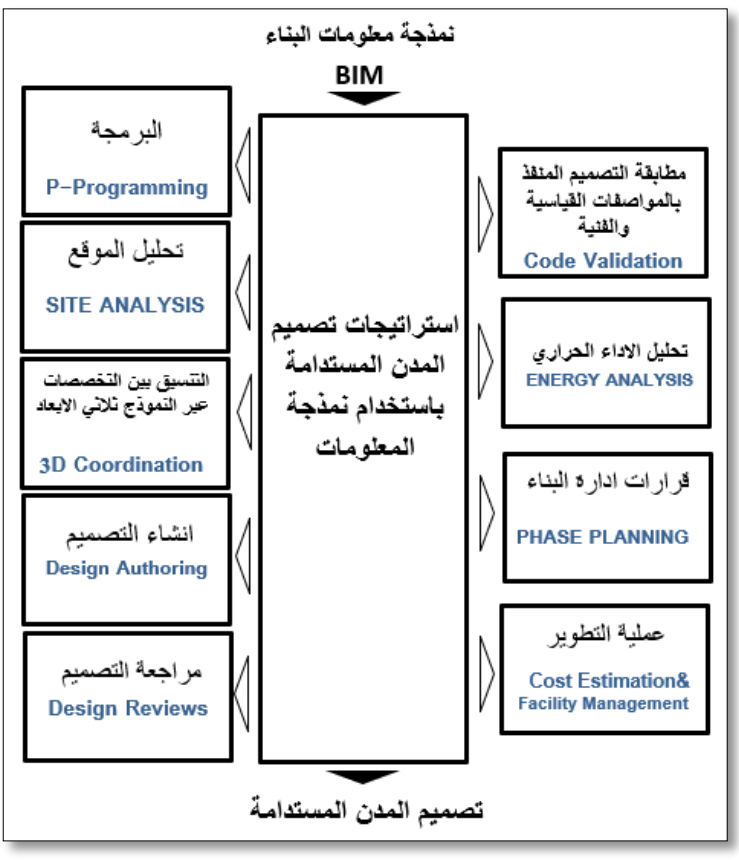

شكل(4) مخطط للنقاط الداعمة لتصميم مدن BIM مستدامة باستخداد

5 - الاستر اتيجية المقترح اتباعها لتصميم مدن مستدامة

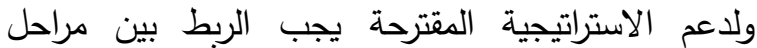

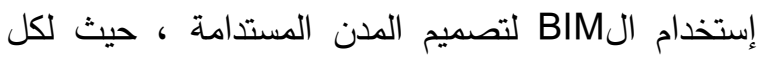

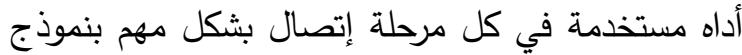
BIM حيث يجب الإنتباه لكل مرحلة لأنها تمثل جزئ فئه

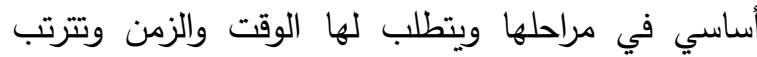
المراحل علي النحو التالي طبقا لترتيبها إحتياجاتها: $P=P L A N$ D=design $C=$ construction $\mathrm{O}=$ operation

1- البرمجة - P-Programming : هي مهام للحفاظ على مرونة المشروع حيث يتم إدخال كافة البيانات والمعلومات الخاصة بالمدينة من تحديد الفراغ الملائم

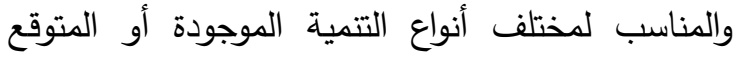

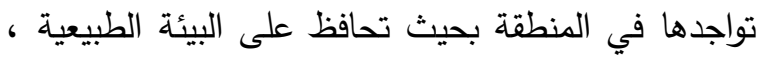
وذلك مساهمة لتأسيس البنية التحتية للمدينة المستدامة بكائة

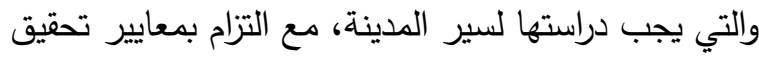
العمارة المستدامة، وهذا الالتزام يشمل جميع مراحل البناء البناء مثل : التخطيط والبناء وإعادة الهيكلة، وكذلك إدارة القضائل التياء والموارد البيئية، مثل الطاقة والمياه و المواد، واستخدام الطاقة بصورة أكثر فاعليه، و أكثر كفاءة. 2-تحليل الموقع SITE ANALYSIS- تحليل ودراسة الموقع العام ، دراسة المناطق المحيطة ومتطلبات تقسيم

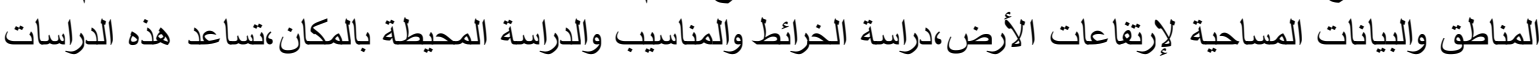

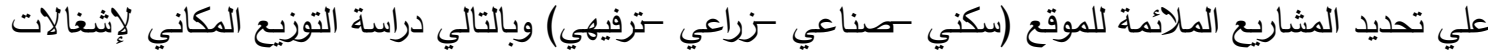

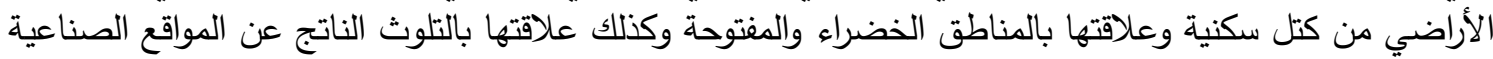

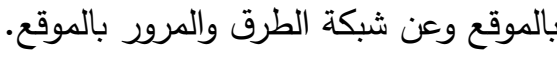

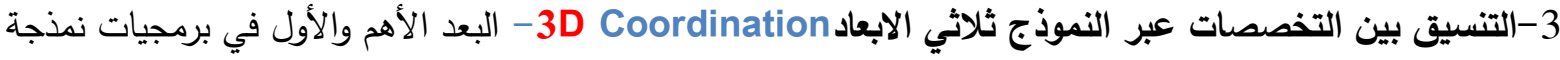

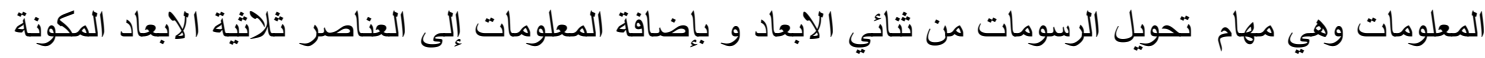

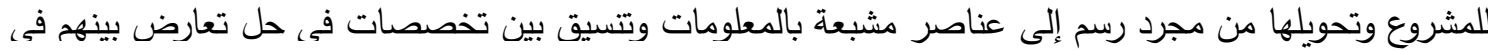

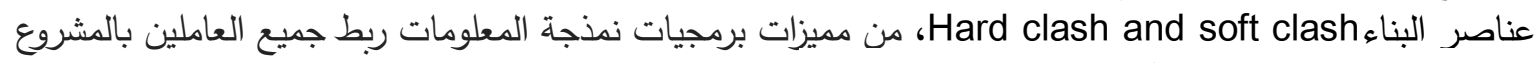
بيعضهم وذلك لتلافي حدوث أي مشاكل تتفيذية بعد ذلك.

4- إنثاء التصميم - Design Authoring- :-عمليه يتم فيها إستخدام نموذج ثلاثي الابعاد لوضع المعلومات التي

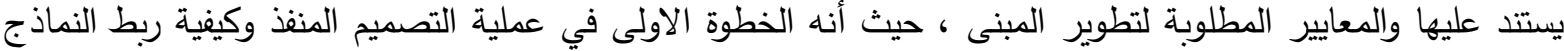
ثلاثية الابعاد مع قاعدة البيانات من كميات مواد الموجودة أساليب وتكاليف البناء, وتسليم نموذج يحتوي على التئ تضاريس 
مساحة البناء وتحليل البيئة المحيطة والمناسيب، وتعتبر تكنولوجيا البيم هي المفتاح لجعل المدن أكثر استدامة وذكاءا،

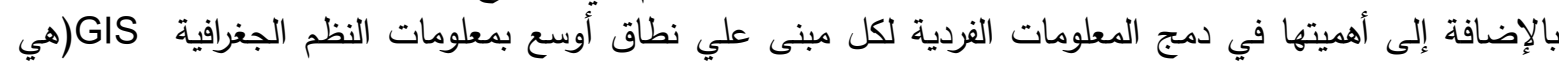
تكنولوجيا مصممة لرصد وتجميع وتحليل كل انواع المعلومات الجغرافية وتمثل نتائج تلك التحاتيات التحليلات

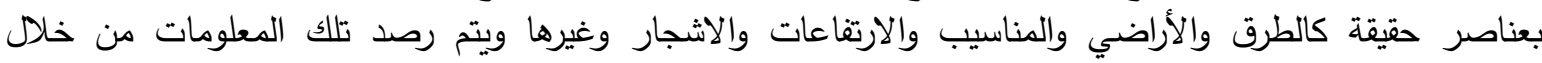

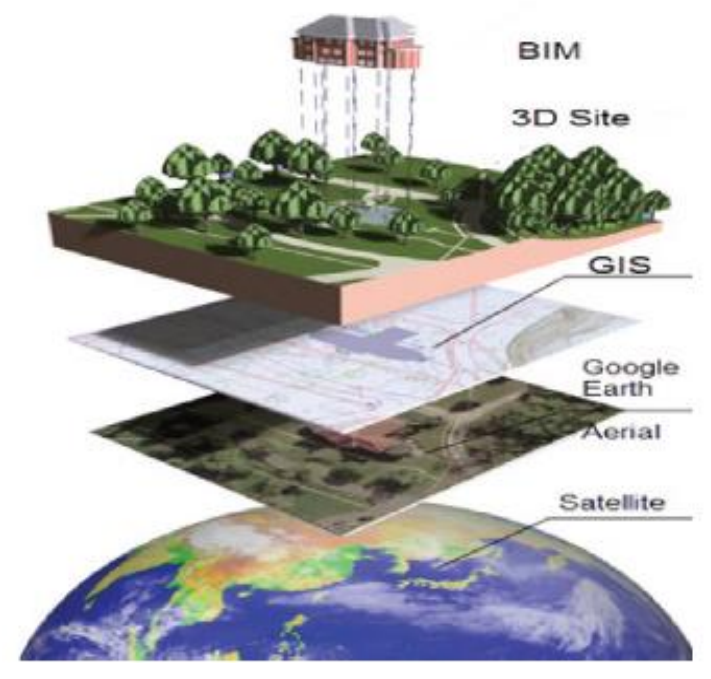

شكل (5) دمج معلومات الخاصة كل مبني بالمعلومات GIS تكنولوجيا الصور الجوية بالأقمار الصناعية والتي تراصيط

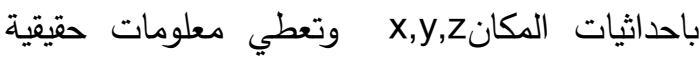

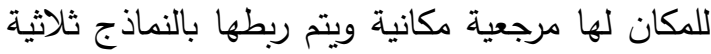
الأبعاد والمواصفات لخلق قاعدة بيم مفتوحة قابلة للتطوي المستمر وربطها بدورة حياة المبنى أو البنات البنية

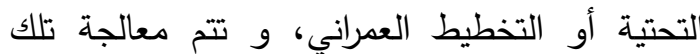

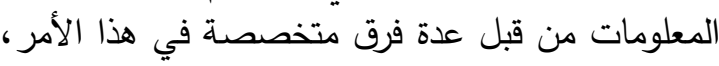

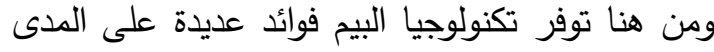

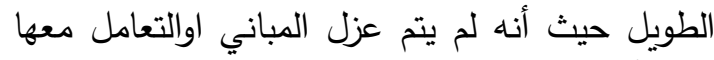

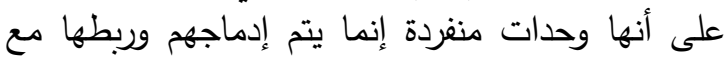

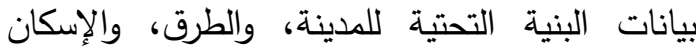

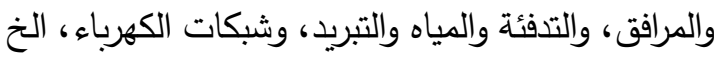
ويسمى ذلك الربط بين بيانات المباني والمدينة بمصنات الكئح

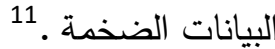

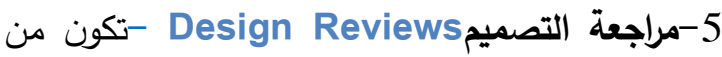
ناحية المصمم لأنه يضع قوانين ومعايير الإضاءة وحل مشاكل التصميم وتقليل الوقت عن طريق حل المشاكل الموجودة

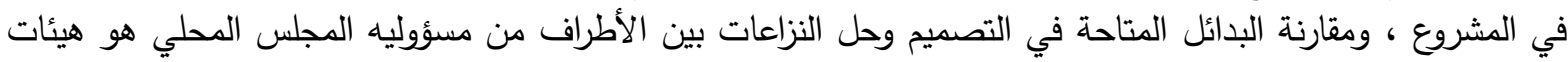

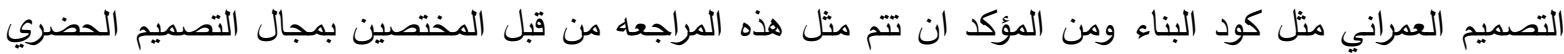

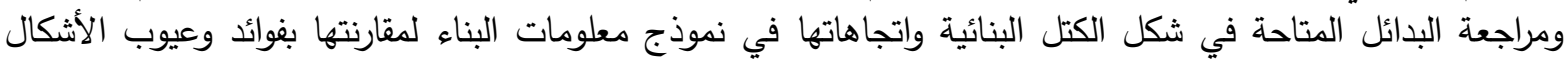
الأخري التي سيتم استتتاجها ل 6- مطابقة التصميم المنفذ بالمواصفات القياسية والفنية Code Validation عملية التحقق من التصميم المنفذ من

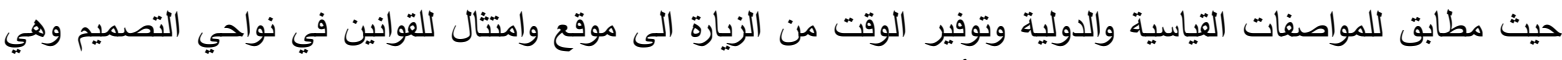

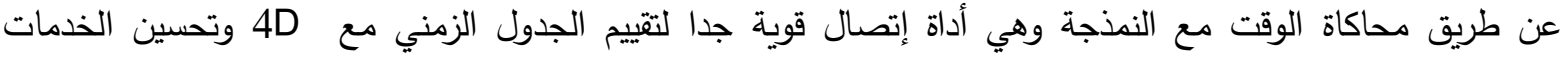

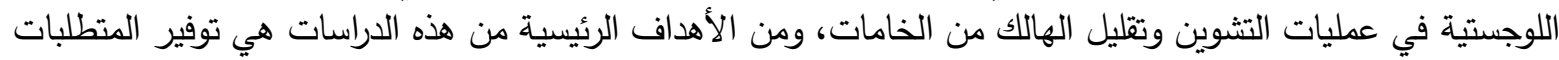
اللازمة لتأمين نظام الحماية من الحرائق ، والتصميم الثامل ،
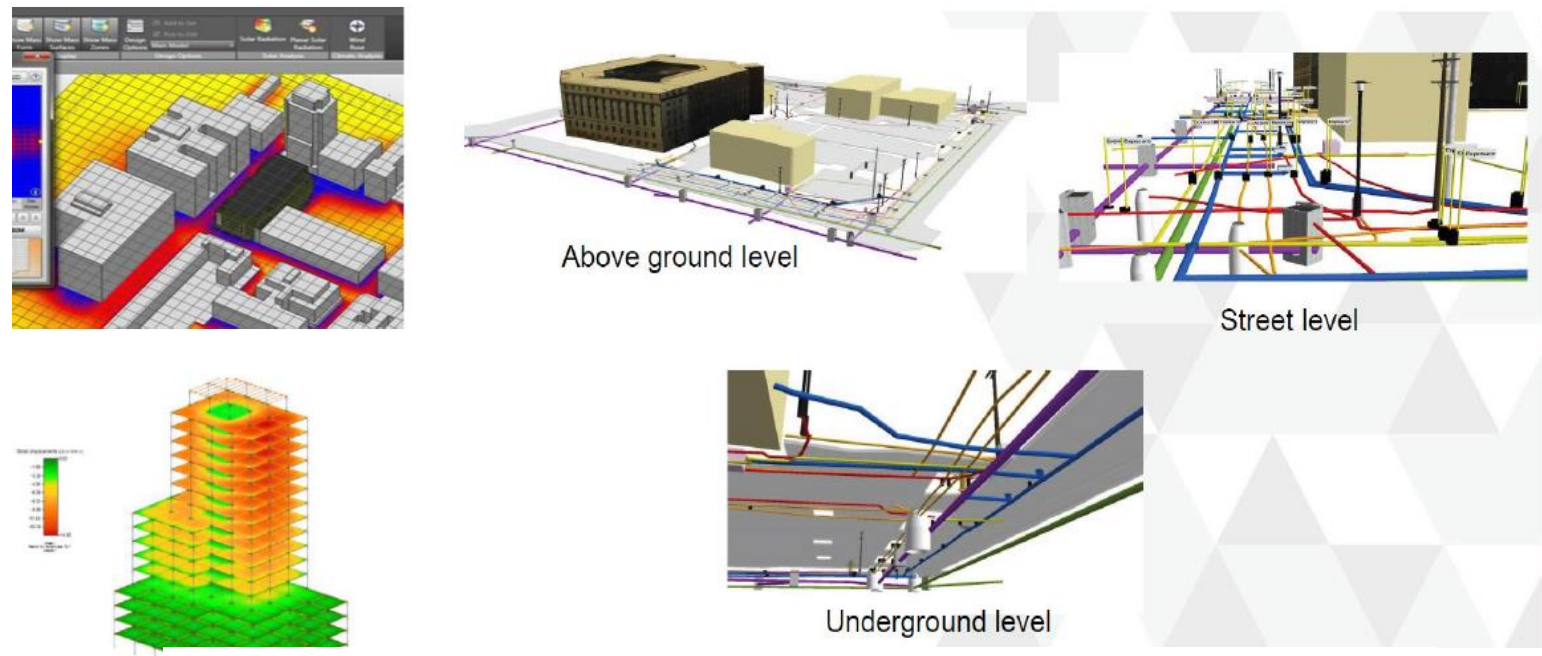

شكل (6) تصميم مراحل المشروعات من خلال ادوات نمذجة معلومات البناء للربط بينهم بالصورة 
النظام البيئي ، الصيانة ،العمليات اليومية ، الترميم ، تحديثات الأعمال الكهربائية والميكانيكية والصحيةMEP، وإدخال

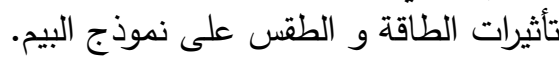

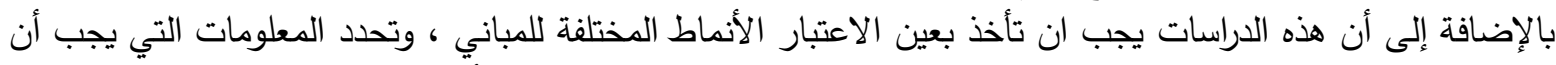

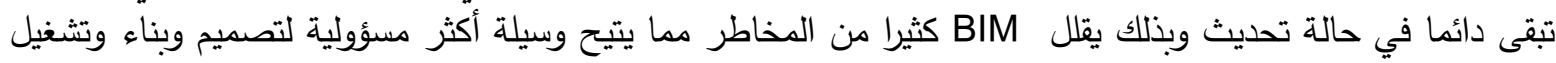
وصيانة الطرق والجسور وأنظمة النقل العام و القدرة على التنبؤ بالتكلفة. 7- تحليل الاداء الحراري -6D-ENERGY ANALYSIS يستند إلى محاكاة المباني وكذلك عناصر الطنة الأنارة ودراسة

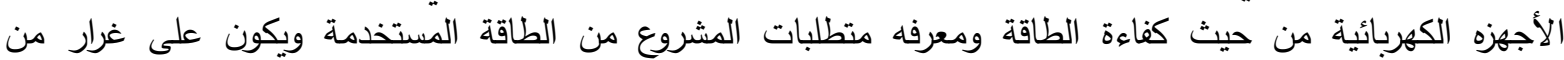

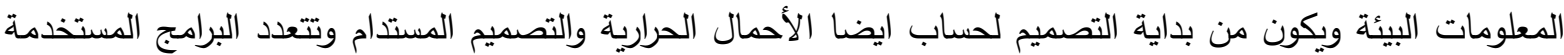

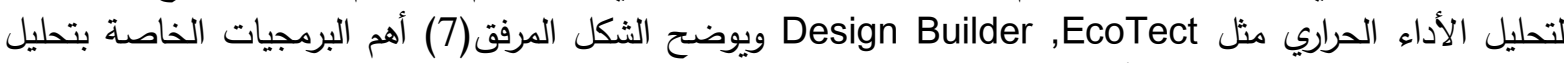
أداء الطاقة وهي الأشهر عالمياء. وبداية عملية التحليل تخلق صورة واضحة لما سيتم قياسه وما هي جوانب التصميم المطلوب تحسينه، يمكن أن يساعد
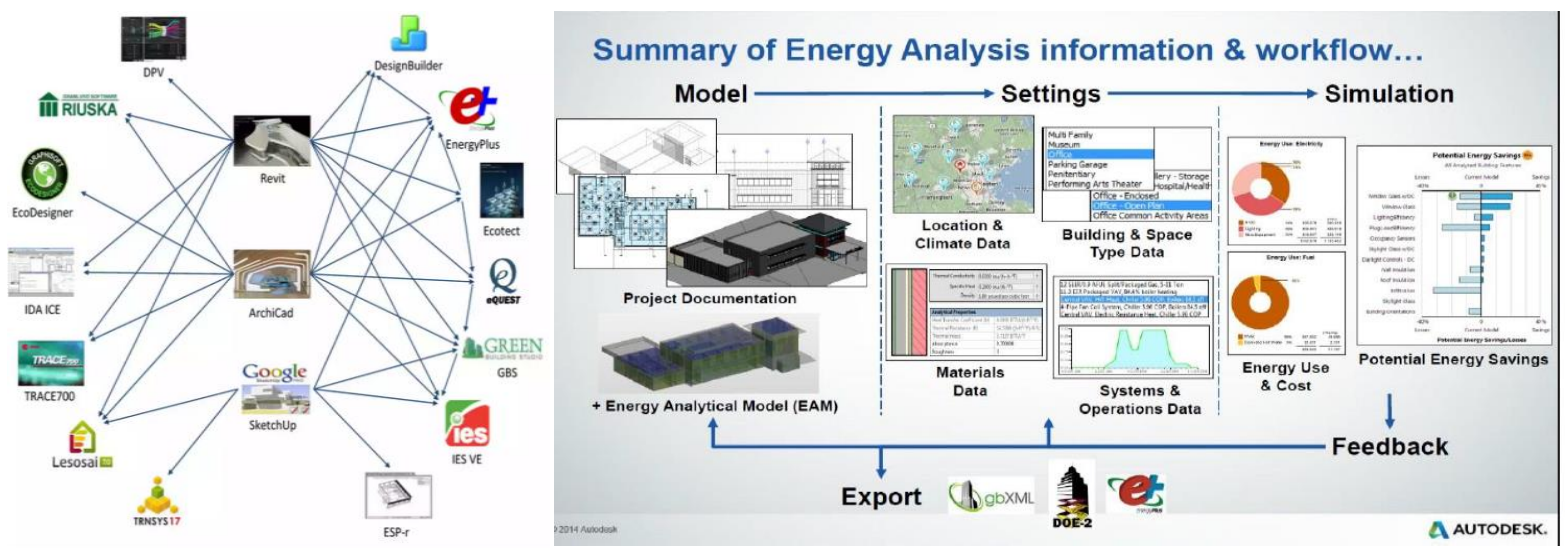

BIM and building analyses و و برامج ال محاكاة الحرارية و تحليل المبني bim (7) تبادل المعلومات بين برامج ال

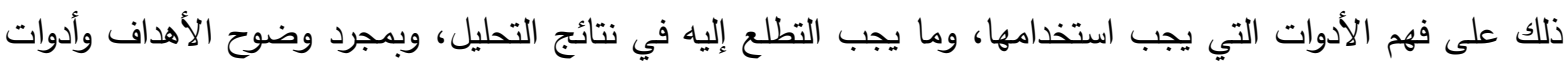

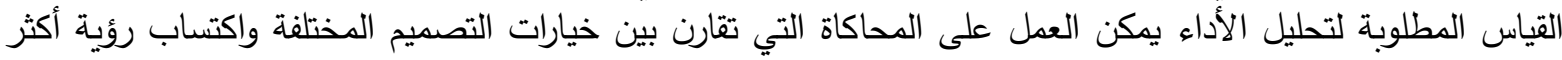
وضوحا من نتائج التحليل؛ مع مراعاة ضرورة توافر بعض الحض الحسابات التقريبية معدة مسبق لقياس مدى منى منطقية نتائج

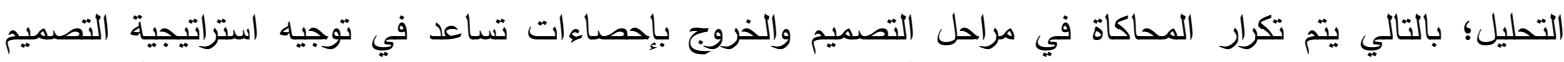

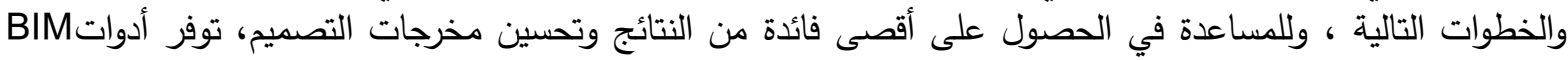

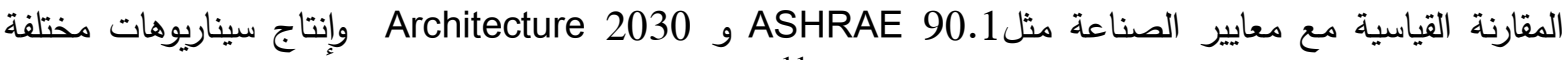

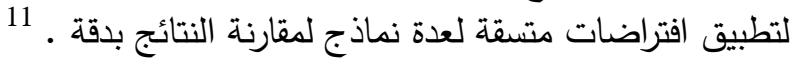
8- قرارات إدارة البناء PHASE PLANNING-يعتمد كليا على 4D يتم معرفة خطوات البناء في الموقع العملية التي

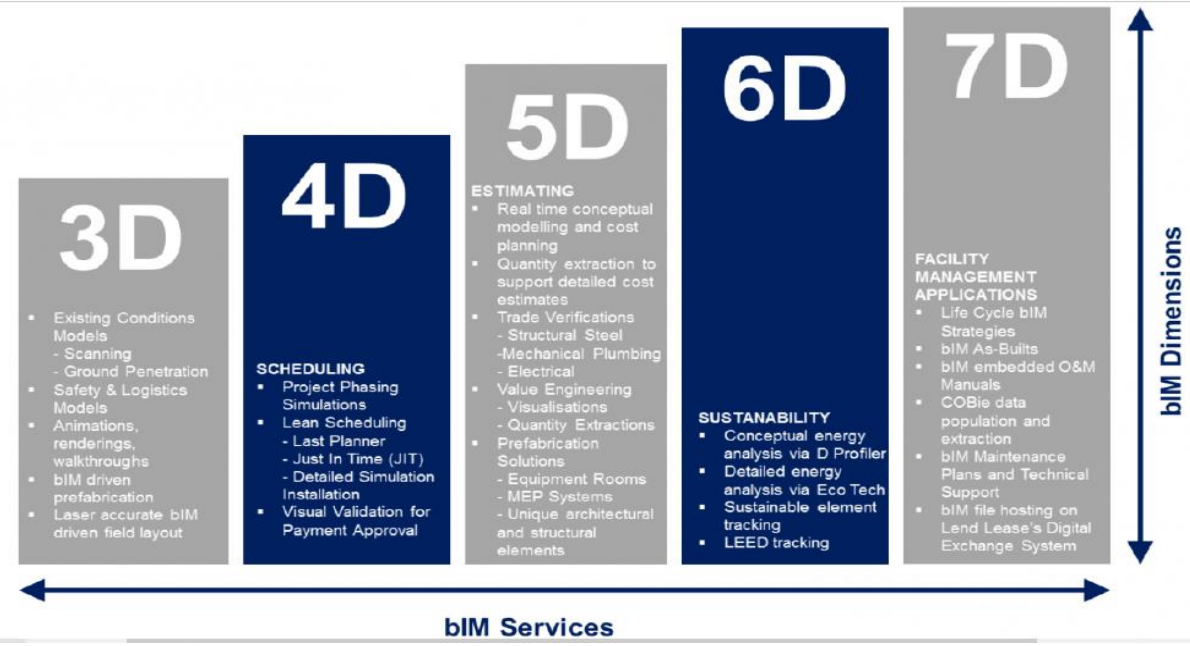
يكون إتجاه

شكل (8) المستويات و الخدمات التي تقدمها برمجيات نمذجة معلومات البناء

الإدارة ذذو إتجاهين ونموذجا قياسيا لزيادة الكفاءة في الصيانة وتشغيل المرافق و و لونائ

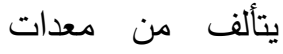
والنظم البيئية المتاحة

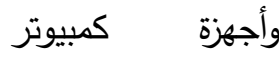
والأعمال الفئنة للشركة حيث تساعد في إتخاذ القرارات المالية وعلى مدى الخىان 
الطويل والتخطيط طويل الددي لكيفيه الحفاظ على المدى قاعدة

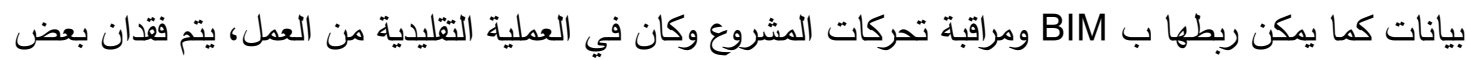

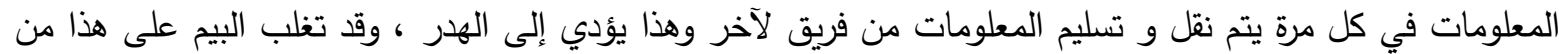

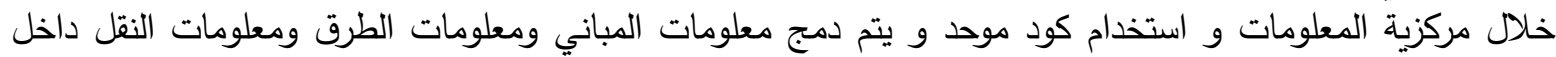

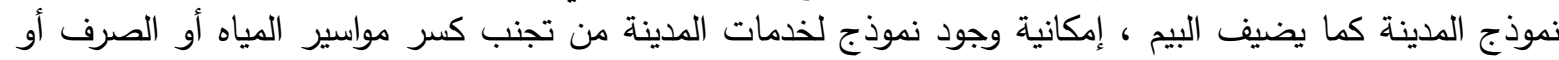

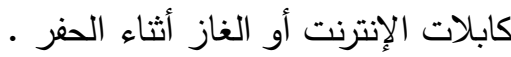

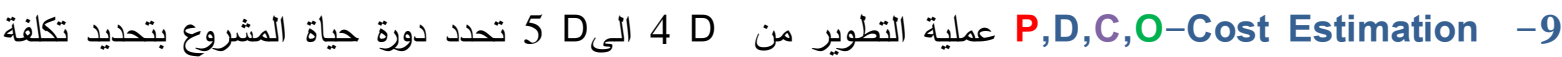
الخامات ومنها تولد كمية الاقراد والمعدات المستخدمة ، والتي تساعد من الحد من التجاوزات في الميزانية وتحديد الأسعار

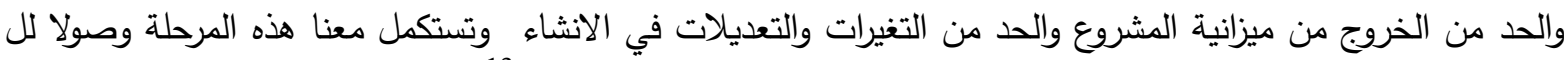

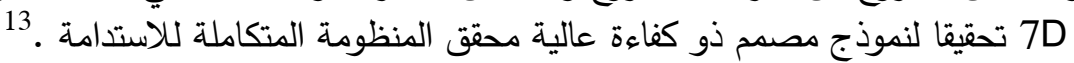

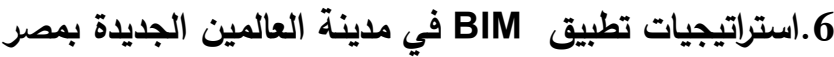

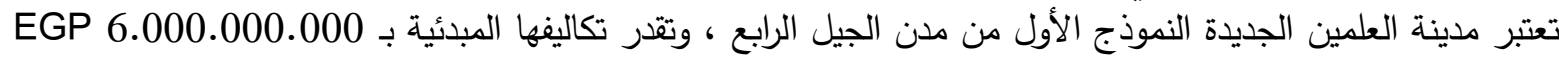

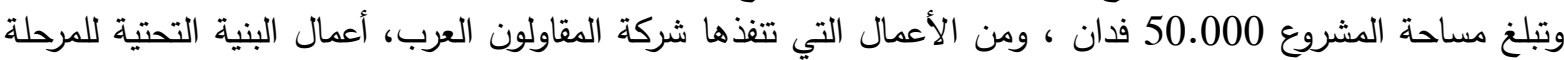

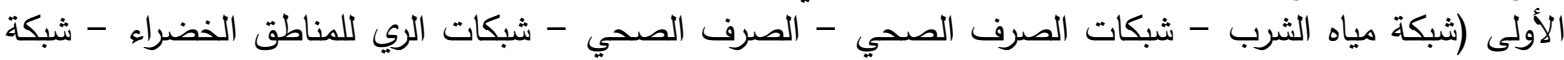

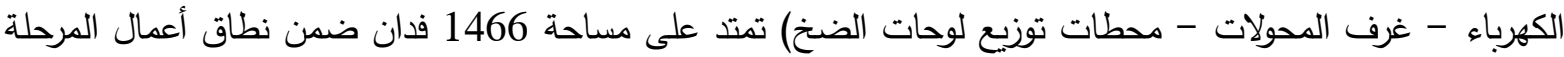

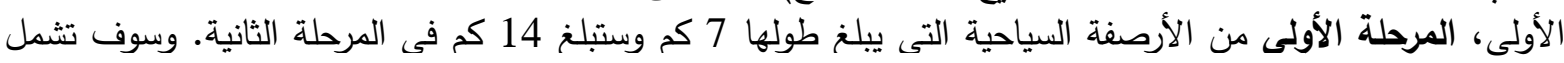

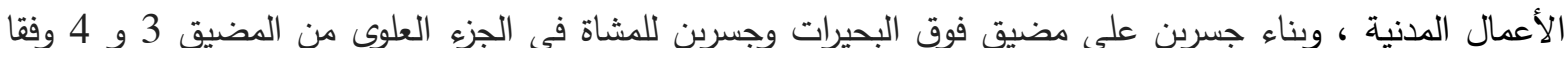

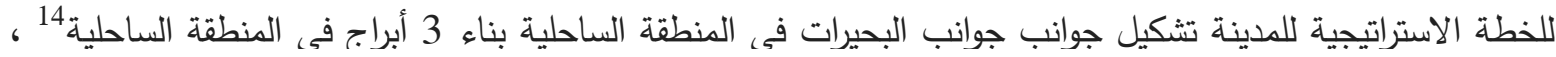

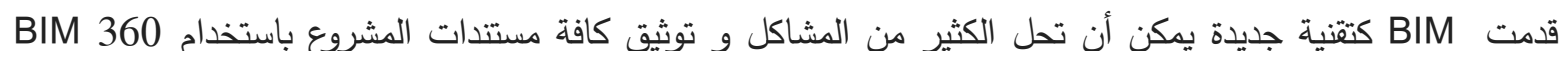
التتسيق بين جميع الرسومات من نفس الانضباط باستخدام Revit ، تقدير التكلفة والجدول الزمني عن طريق

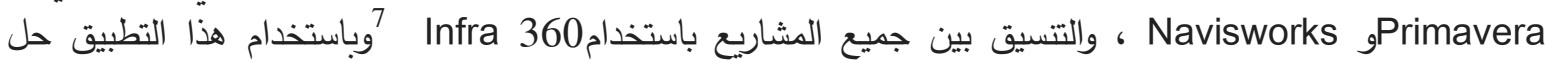

الكثير من المشاكل:

- تتظيم الرسومات والوثائق عن طريق القراءة تلقائيا باستخدام الرموز •

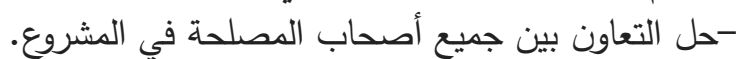

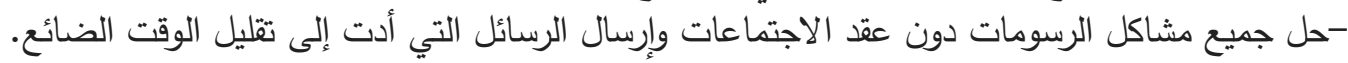

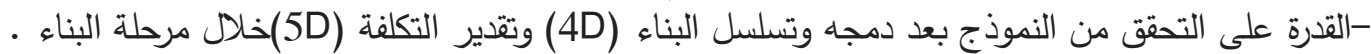

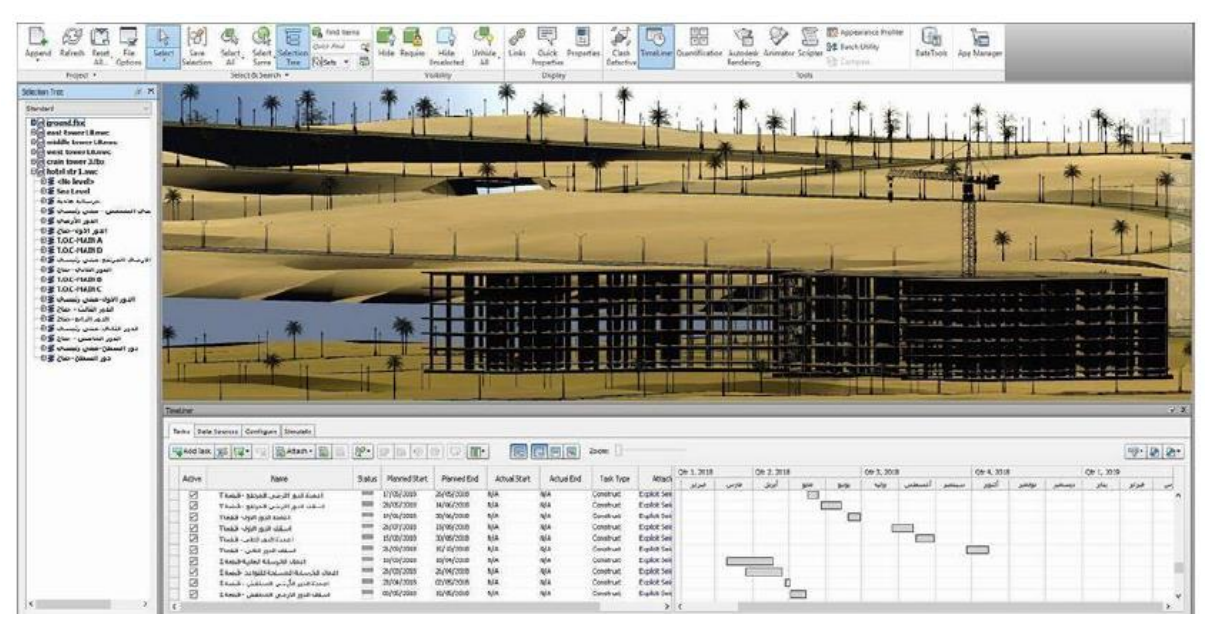

شكل (9) انثاء الابر اج السكنية القوات المسلحة منطقة العالمين 


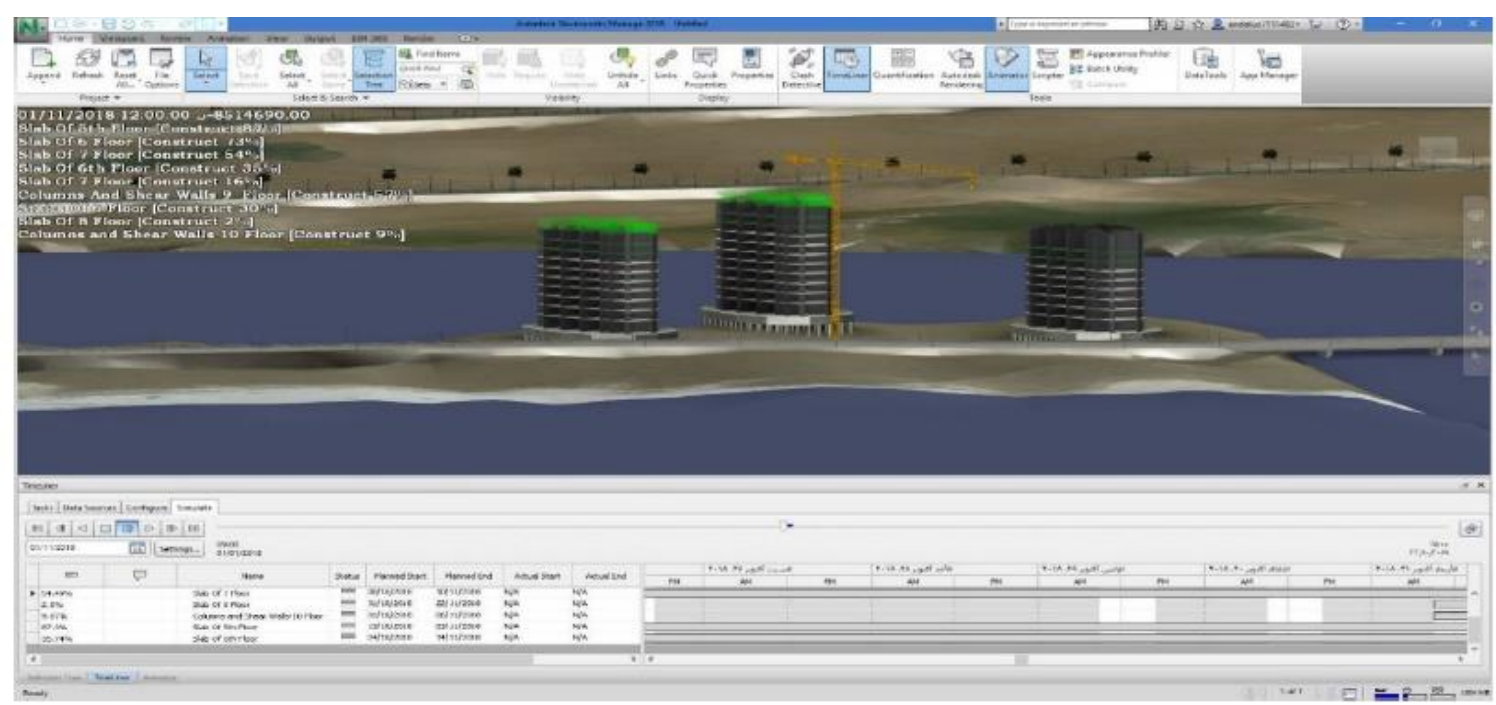

شكل (10) انشاء الابر اج السكنية باستخدام برمجيات نمذجة معلومات البناء BIM

- استخدام تطبيق INFRA WORKS 360 والذي يسمح بفهم سير العملية التخطيطية، كما يمكن من خلال المحاكاة البصرية المقارنة بين المخطط تنفيذه والجزه الفعلي وذلك للتسيق بين المشاريع في البيئة الافتراضية والتحقق من

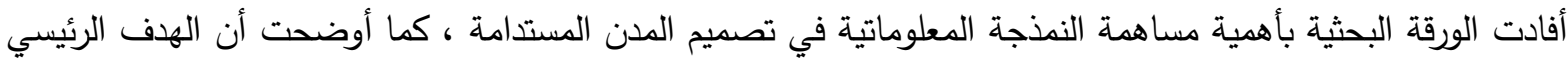

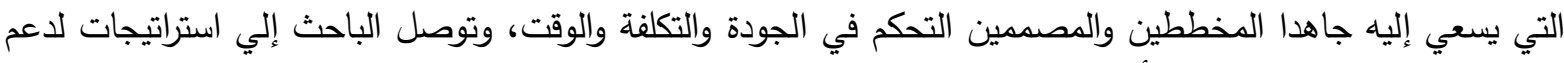
خطة الدولة المستقبلية والتي بدأت في خطواتها السليمة في بناء مدن مستدامة وذلك من خلال التالك استخدام

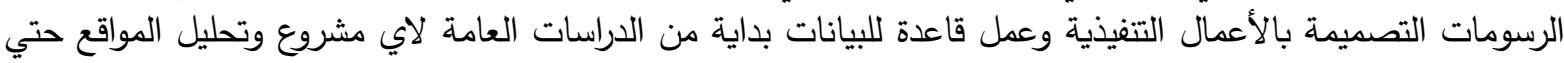

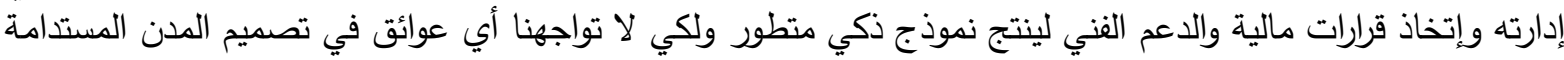

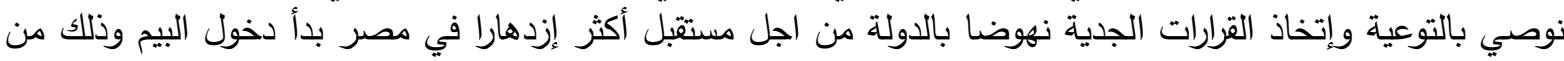
خلال:

1- التعاون بين مهندسي المشروع وأصحاب المصلحة والتتسيق والتكامل بين الرسومات والذي سيحقق تقليل الوقت

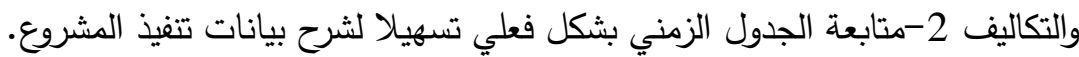

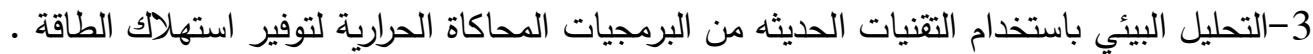

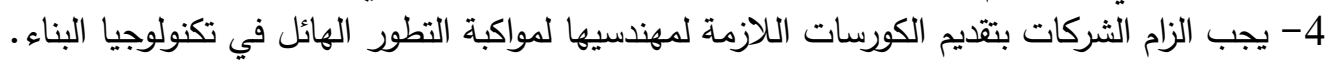
5-الإستعانه بالمهندسين المتخصصين في هذه البرمجيات لتنفيذ اللمشروعات القومية الجديدة. وسوف تساهم تطبيق الاستراتيجيات المقترحة بشكل كبير في تصميم مدن مستدامة يمشي علي نهج تصميمها جميع المدن المستدامة والموفره للطاقة لاحقا.

Abstract

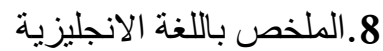

The use of the applications of modern technology and digital models in architecture has moved from design to advanced stages of architectural innovation. Recently, more sophisticated tools have emerged that represent the building as an integrated database of coordinated information. It is completely different from the traditional use of the software called Building Information Modeling (BIM), The adoption of the BIM model and its potentials has been demonstrated by the progress and success achieved by the implementation of the BIM model and its potentials which have enabled the construction industry to become more sophisticated in the areas of planning, design, implementation, operation and management. Of the development of the tools and means of production of the building in a way that ensures improvement of the level of quality and organization and communication between employees at all stages of the project in addition to reducing time and cost. 
The country has taken the right path in recent years to set up a number of national projects and create completely different cities from traditional cities. Design and construction based on modern technologies in the field of computer. There are initiatives at the national level to produce BIM standards and guidelines. The research aims at implementing a strategy and methodology for the implementation of sustainable green cities through documenting and highlighting the role of modern applications in the field of computer and its impact on the quality of the product. For the architect.

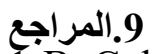

1-B. Cohen, "Urbanization in developing countries: Current trends, future projections, and key challenges for sustainability," Technol. Soc., vol. 28, no. 1, pp. 63-80, 2006.

2-Abolsaud, A. A. \&. Y., 2018. Integrating BIM-based Simulation Technique for Sustainable Building Design. Cairo, SPRINGER.

3-AECOM, D. G. B., 2017. AFRICA PROPERTY \& CONSTRUCTION COST GUIDE 2017, South Africa: AECOM SA (Pty) Limited.

4-Azhar, S., 2011. Building Information Modeling (BIM): Trends, Benefits, Risks, and Challenges for the AEC Industry. Leadership Manage. Eng., 11.

5-http://gate.ahram.org.eg/News/1828277.aspx

6- Paul Doherty, President \& CEO, The Digit Group, Inc., http://www.thedigitgroupinc.com/

7-https//www.construction.com/toolkit/reports/the-business-value-of-BIM-forinfrastructure 2017

8-Aksamija, A., 2015. A STRATEGY FOR ENERGY PERFORMANCE ANALYSIS AT THE EARLY DESIGN STAGE: PREDICTED VS. ACTUAL BUILDING ENERGY PERFORMANCE. Journal of Green Building, 10(No. 3), p. pp. 161176.

9-Analysts, L., 2018. [Online] Available at: https://www.agi32.com/ [Accessed 2018]. Available https://knowledge.autodesk.com/searchresult/caas/CloudHelp/cloudhelp/ENU/BPA.Perform anceStudies/files/GUID-5AE6A81D-B170-47A5-8BF8-190235F25287-htm.html [Accessed 2018]. Available at: http://blogs.autodesk.com/insight/leed-sda-ase-studies-with-insightrevit-2017-plugin/ [Accessed 2703 2018].

10-Autodesk, U., 2017. Autodesk Workshop Sustainability, s.1.: Autodesk University.

11-Ebrahim P. Karan, J. I. a. J. H., 2015. BIM and GIS Integration and Interoperability Based on Semantic Web Technology. Journal of Computing in Civil Engineering.

12-Energy, U. D. o., 2006. Sustainable Building Rating Systems, s.1.: U.S. Department of Energy - General Services Administration.

13-M. Senave, S. B., 2015. Building Information Modelling (BIM) in Design, Construction and Operations. WIT Press, p. 351.

14-Eng Islam mohamed khalil -The Implementation of BIM in the New AlAmein city -Egypt $1 / 2018$. 